\title{
Features of the Extension of a Statistical Measure of Complexity to Continuous Systems
}

\author{
Raquel G. Catalán*, José Garay ${ }^{\dagger}$ and Ricardo López-Ruiz \\ * Dpto. de Matemáticas e Informática, \\ Universidad Pública de Navarra, 31008-Pamplona, Spain. \\ † Departamento de Matemáticas, \\ ‡ DIIS - Área de Ciencias de la Computación, \\ Facultad de Ciencias, Edificio B, \\ Universidad de Zaragoza, 50009-Zaragoza, Spain.
}

date: October-2001

\begin{abstract}
We discuss some aspects of the extension to continuous systems of a statistical measure of complexity introduced by López-Ruiz, Mancini and Calbet (LMC) [Phys. Lett. A 209 (1995) 321]. In general, the extension of a magnitude from the discrete to the continuous case is not a trivial process and requires some choice. In the present study, several possibilities appear available. One of them is examined in detail. Some interesting properties desirable for any magnitude of complexity are discovered on this particular extension.
\end{abstract}

Keywords: Complexity; Entropy; Disequilibrium; Continuous systems; Invariance properties. e-mail: rilopez@posta.unizar.es 


\section{Introduction}

In the last years many complexity measures have been proposed as indicators of the complex behaviour found in different systems scattered in a broad spectrum of fields. Some of them come from physics such as the effective measure of complexity [1], the thermodynamical depth [2] and the simple measure of complexity [3]. Other attempts arise from the field of computational sciences such as algorithmic complexity [4, [5], Lempel-Ziv complexity [6] and $\epsilon$-machine complexity [7]. Other works try to enlighten this question in many other contexts: ecology, genetics, economy, etc., for instance, the complexity of a system based on its diversity [8] and the physical complexity of genomes [9].

Most of these proposals coincide in using concepts such as entropy (in physics) or information (in computational sciences) as a basic ingredient for quantifying the complexity of a phenomenon. Also there is a general belief that the notion of complexity in physics must start by considering the perfect crystal and the isolated ideal gas as examples of simple models with zero complexity. Both systems are extrema in an entropy/information scale and therefore some fundamental ingredient would be missing if one insisted on describing complexity only with the ordinary information or entropy.

It seems reasonable to adopt some kind of distance to the equiparti- 
tion, the disequilibrium of the system, as a new ingredient for defining an indicator of complexity. Going back to the two former examples, it is readily seen that they are extremes in a disequilibrium scale and therefore disequilibrium cannot be directly associated with complexity.

The recently introduced López-Ruiz-Mancini-Calbet (LMC) statistical measure of complexity [10] identifies the entropy or information stored in a system and its distance to the equilibrium probability distribution (the disequilibrium) as the two ingredients giving the correct asymptotic properties of a well-behaved measure of complexity. In fact, it vanishes both for completely ordered and for completely random systems. Besides giving the main features of an intuitive notion of complexity, it has been shown that LMC complexity successfully enables us to discern situations regarded as complex in discrete systems out of equilibrium: one instance of a local transition to chaos via intermittency in the logistic map 10, the dynamical behaviour of this quantity in a simplified isolated gas [11] and another example of classical statistical mechanics [12].

A possible formula of LMC complexity for continuous systems was suggested in López-Ruiz et al. [10]. Anteneodo and Plastino [13] pointed out some peculiarities concerning such an extension for continuous probability distributions. It is the aim of this work to offer a discussion of the extension of LMC complexity for continuous systems. A slightly modified extension brings interesting and very striking properties, and some 
of the Anteneodo and Plastino remarks are solved with the new proposed definition.

In Section 2 the extension of information and disequilibrium concepts for the continuous case are discussed. In Section 3 the LMC measure of complexity is rewieved and possible extensions for continuous systems are suggested. We proceed to present some properties of one of these extensions in Section 4. Finally, we establish the Conclusions.

\section{Entropy/Information and Disequilibrium}

Depending on the necessary conditions to fulfill, the extension of an established formula from the discrete to the continuous case always requires a careful study and in many situations some kind of choice between several possibilities. Next we carry out this process for the entropy and disequilibrium formulas.

\subsection{Entropy or Information}

Given a discrete probability distribution $\left\{p_{i}\right\}_{i=1,2, \ldots, N}$ satisfying $p_{i} \geq 0$ and $\sum_{i=1}^{N} p_{i}=1$, the Boltzmann-Gibss-Shannon formula 14 that accounts for the entropy or information, $H$, stored in a system is defined 
by

$$
H\left(\left\{p_{i}\right\}\right)=-k \sum_{i=1}^{N} p_{i} \log p_{i},
$$

where $k$ is a positive constant. Some properties of this quantity are: (i) positivity: $H \geq 0$ for any arbitrary set $\left\{p_{i}\right\}$, (ii) concavity: $H$ is concave for arbitrary $\left\{p_{i}\right\}$ and reaches the extremal value for equiprobability $\left(p_{i}=\right.$ $1 / N \forall i)$, (iii) additivity: $H(A \cup B)=H(A)+H(B)$ where $A$ and $B$ are two independent systems, and (iv) continuity: $H$ is continuous for each of its arguments. And vice versa, it has been shown that the only function of $\left\{p_{i}\right\}$ verifying the latter properties is given by Eq. (11) [14, 15]. For an isolated system, the irreversibility property is also verified, that is, the time derivative of $H$ is positive, $d H / d t \geq 0$, reaching the equality only for equilibrium.

Calculation of $H$ for a continuous probability distribution $p(x)$, with support on $[-L, L]$ and $\int_{-L}^{L} p(x) d x=1$, can be performed by dividing the interval $[-L, L]$ in small equal-length pieces $\Delta x=x_{i}-x_{i-1}, i=$ $1, \cdots, n$, with $x_{0}=-L$ and $x_{n}=L$, and by considering the approximated discrete distribution $\left\{p_{i}\right\}=\left\{p\left(\bar{x}_{i}\right) \Delta x\right\}, i=1, \cdots, n$, with $\bar{x}_{i}$ a point in the segment $\left[x_{i-1}, x_{i}\right]$. It gives us

$$
\begin{aligned}
H^{*} & =H\left(\left\{p_{i}\right\}\right)= \\
& =-k \sum_{i=1}^{n} p\left(\bar{x}_{i}\right) \log p\left(\bar{x}_{i}\right) \Delta x-k \sum_{i=1}^{n} p\left(\bar{x}_{i}\right) \log (\Delta x) \Delta x .
\end{aligned}
$$

The second adding term of $H^{*}$ in the expression (2) grows as $\log n$ when $n$ goes to infinity. Therefore it seems reasonable to take just the first and 
finite adding term of $H^{*}$ as the extension of $H$ to the continuous case: $H(p(x))$. It characterizes with a finite number the information contained in a continuous distribution $p(x)$. In the limit $n \rightarrow \infty$, we obtain

$$
\begin{aligned}
H(p(x)) & =\lim _{n \rightarrow \infty}\left[-k \sum_{i=1}^{n} p\left(\bar{x}_{i}\right) \log p\left(\bar{x}_{i}\right) \Delta x\right]= \\
& =-k \int_{-L}^{L} p(x) \log p(x) d x .
\end{aligned}
$$

If $p(x) \geq 1$ in some region, the entropy defined by Eq. (3) can become negative. Although this situation is mathematically possible and coherent, it is unfounded from a physical point of view. See [16 for a discussion on this point. Let $f(p, q)$ be a probability distribution in phase space with coordinates $(p, q), f \geq 0$ and $d p d q$ having the dimension of an action. In this case the volume element is $d p d q / h$ with $h$ the Planck constant. Suppose that $H(f)<0$. Because of $\int(d p d q / h) f=1$, the extent of the region where $f>1$ must be smaller than $h$. Hence a negative classical entropy arises if one tries to localize a particle in phase space in a region smaller than $h$, that is, if the uncertainty relation is violated. In consequence, not every classical probability distribution can be observed in nature. The condition $H(f)=0$ could give us the mininal width that is physically allowed for the distribution and so the maximal localization of the system under study. This cutting property has been used in the calculations performed in Ref. [12]. 


\subsection{Disequilibrium}

Given a discrete probability distribution $\left\{p_{i}\right\}_{i=1,2, \ldots, N}$ satisfying $p_{i} \geq 0$

and $\sum_{i=1}^{N} p_{i}=1$, its Disequilibrium, $D$, is the quadratic distance of the actual probability distribution $\left\{p_{i}\right\}$ to equiprobability:

$$
D\left(\left\{p_{i}\right\}\right)=\sum_{i=1}^{N}\left(p_{i}-\frac{1}{N}\right)^{2} .
$$

$D$ is maximal for fully regular systems and vanishes for completely random ones.

In the continuous case with support on the interval $[-L, L]$, the rectangular function $p(x)=1 /(2 L)$, with $-L<x<L$, is the natural extension of the equiprobability distribution of the discrete case. The disequilibrium could be defined as

$$
D^{*}=\int_{-L}^{L}\left(p(x)-\frac{1}{2 L}\right)^{2} d x=\int_{-L}^{L} p^{2}(x) d x-\frac{1}{2 L} .
$$

If we redefine $D$ omitting the constant adding term in $D^{*}$, the disequilibrium reads now:

$$
D(p(x))=\int_{-L}^{L} p^{2}(x) d x .
$$

$D>0$ for every distribution and it is minimal for the rectangular function which represents the equipartition. $D$ does also tend to infinity when the width of $p(x)$ narrows strongly and becomes extremely peaked. 


\section{$3 \quad$ Statistical Measure of Complexity}

LMC complexity, $C$, has been defined [10] as the interplay between the information, $H$, stored in a system and its disequilibrium, $D$. Calculation

of $C$ for a discrete distribution $\left\{p_{i}\right\}$, with $p_{i} \geq 0$ and $i=1, \cdots, N$, is given by the formula

$$
\begin{aligned}
C\left(\left\{p_{i}\right\}\right) & =H\left(\left\{p_{i}\right\}\right) \cdot D\left(\left\{p_{i}\right\}\right)= \\
& =-k\left(\sum_{i=1}^{N} p_{i} \log p_{i}\right) \cdot\left(\sum_{i=1}^{N}\left(p_{i}-\frac{1}{N}\right)^{2}\right) .
\end{aligned}
$$

This definition fits the intuitive arguments and verifies the required asymptotic properties: it vanishes for completely ordered systems and for fully random systems. $C$ has been successfully calculated in different systems out of equilibrium: one instance of a local transition to chaos in an unidimensional mapping [10], the time evolution of $C$ for a simplified model of an isolated gas, the 'tetrahedral' gas [11], some statistical features of the behaviour of LMC complexity for DNA sequences [17] and a modification of $C$ as an effective method to identify the complexity in hydrological systems 18 .

Feldman and Cruchtfield [19 presented as a main drawback that $C$ vanishes and it is not an extensive variable for finite-memory regular Markov chains when the system size increases. This is not the general behaviour of $C$ in the thermodynamic limit as it has been suggested by Calbet and López-Ruiz [11]. On the one hand, when $N \rightarrow \infty$ and 
$k=1 / \log N$, LMC complexity is not a trivial function of the entropy, in the sense that for a given $H$ there exists a range of complexities between 0 and $C_{\max }(H)$, where $C_{\max }$ is given by

$$
\left[C_{\max }(H)\right]_{N \rightarrow \infty}=H \cdot(1-H)^{2}
$$

Observe that in this case $H$ is normalized, $0<H<1$, because $k=$ $1 / \log N$. On the other hand, non-extensitivity cannot be considered as an obstacle since it is nowadays well known that there exists a variety of physical systems for which the classical statistical mechanics seems to be inadequate and for which an alternative non-extensive thermodynamics is being hailed as a possible basis of a theoretical framework appropriate to deal with them [20].

According to the discussion in Section 2, the expression of $C$ for the case of a continuum number of states, $x$, with support on the interval $[-L, L]$ and $\int_{-L}^{L} p(x) d x=1$, is defined by

$$
\begin{aligned}
C(p(x)) & =H(p(x)) \cdot D(p(x))= \\
& =\left(-k \int_{-L}^{L} p(x) \log p(x) d x\right) \cdot\left(\int_{-L}^{L} p^{2}(x) d x\right) .
\end{aligned}
$$

Anteneodo and Plastino [13 pointed out that $C$ can become negative. Obviously, $C<0$ implies $H<0$. Although this situation is coherent from a mathematical point of view, it is not physically possible. Hence a negative entropy means to localize a system in phase space into a region smaller than $h$ (Planck constant) and this would imply to violate 
the uncertainty principle (see discussion of Section 2.11). Then a distribution can broaden without any limit but it cannot become extremely peaked. The condition $H=0$ could indicate the minimal width that $p(x)$ is allowed to have. Similarly to the discrete case, $C$ is positive for any situation and vanishes both for an extreme localization and for the most widely delocalization embodied by the equiprobability distribution. Thus, LMC complexity can be straightforwardly calculated for any continuous distribution by Eq. (8). It has been applied, for instance, for quantifying $C$ in a simplified two-level laser model in Ref. [12].

Anyway, the positivity of $C$ for every distribution in the continuous case can be recovered by taking the exponential of $H$. If we define $\hat{H}=$ $e^{H}$, we obtain a new expression $\hat{C}$ of the statistical measure of complexity given by

$$
\hat{C}(p(x))=\hat{H}(p(x)) \cdot D(p(x))=e^{H(p(x))} \cdot D(p(x)) .
$$

In addition to the positivity, $\hat{C}$ encloses other interesting properties that we describe in the next section.

\section{Properties of $\hat{C}$}

The quantity $\hat{C}$ given by Eq. (9) has been presented as one of the possible extensions of the LMC complexity for continuous systems. We proceed 
now to present some of the properties that characterize such a complexity indicator.

\subsection{Invariance under translations and rescaling trans- formations}

If $p(x)$ is a density function defined on the real axis $\mathbf{R}, \int_{\mathbf{R}} p(x) d x=1$, and $\alpha>0$ and $\beta$ are two real numbers, we denote by $p_{\alpha, \beta}(x)$ the new probability distribution obtained by the action of a $\beta$-translation and an $\alpha$-rescaling transformation on $p(x)$,

$$
p_{\alpha, \beta}(x)=\alpha p(\alpha(x-\beta)) .
$$

When $\alpha<1, p_{\alpha, \beta}(x)$ broadens whereas if $\alpha>1$ it becomes more peaked. Observe that $p_{\alpha, \beta}(x)$ is also a density function. After making the change of variable $y=\alpha(x-\beta)$ we obtain

$$
\int_{\mathbf{R}} p_{\alpha, \beta}(x) d x=\int_{\mathbf{R}} \alpha p(\alpha(x-\beta)) d x=\int_{\mathbf{R}} p(y) d y=1 .
$$

The behaviour of $H$ under the transformation given by Eq. (10) is the following:

$$
\begin{aligned}
H\left(p_{\alpha, \beta}\right) & =-\int_{\mathbf{R}} p_{\alpha, \beta}(x) \log p_{\alpha, \beta}(x) d x=-\int_{\mathbf{R}} p(y) \log (\alpha p(y)) d y \\
& =-\int_{\mathbf{R}} p(y) \log p(y) d y-\log \alpha \int_{\mathbf{R}} p(y) d y \\
& =H(p)-\log \alpha .
\end{aligned}
$$

Then,

$$
\hat{H}\left(p_{\alpha, \beta}\right)=e^{H\left(p_{\alpha, \beta}\right)}=\frac{\hat{H}(p)}{\alpha} .
$$


It is straightforward to see that $D\left(p_{\alpha, \beta}\right)=\alpha D(p)$, and to conclude that

$$
\hat{C}\left(p_{\alpha, \beta}\right)=\hat{H}\left(p_{\alpha, \beta}\right) \cdot D\left(p_{\alpha, \beta}\right)=\frac{\hat{H}(p)}{\alpha} \alpha D(p)=\hat{C}(p) .
$$

Observe that translations and rescaling transformations keep also the shape of the distributions. Then it could be reasonable to denominate the invariant quantity $\hat{C}$ as the shape complexity of the family formed by a distribution $p(x)$ and its transformed $p_{\alpha, \beta}(x)$. Hence, for instance, the rectangular $\Pi(x)$, the isosceles-triangle shaped $\Lambda(x)$, the gaussian $\Gamma(x)$, or the exponential $\Xi(x)$ distributions continue to belong to the same $\Pi$, $\Lambda, \Gamma$ or $\Xi$ family, respectively, after applying the transformations defined by Eq. (10). Calculation of $\hat{C}$ on these distribution families gives us

$$
\begin{aligned}
& \hat{C}(\Pi)=1 \\
& \hat{C}(\Lambda)=\frac{2}{3} \sqrt{e} \approx 1.0991 \\
& \hat{C}(\Gamma)=\sqrt{\frac{e}{2}} \approx 1.1658 \\
& \hat{C}(\Xi)=\frac{e}{2} \approx 1.3591 .
\end{aligned}
$$

Remark that the family of rectangular distributions has a smaller $\hat{C}$ than the rest of distributions. This fact is true for every distribution and it will be proved in Section 4.4 .

\subsection{Invariance under replication}

Lloyd and Pagels [2] recommend that a complexity measure should remain essentially unchanged under replication. We show now that $\hat{C}$ is 
replicant invariant, that is, the shape complexity of $m$ replicas of a given distribution is equal to the shape complexity of the original one.

Suppose $p(x)$ a compactly supported density function, $\int_{-\infty}^{\infty} p(x) d x=$ 1. Take $n$ copies $p_{m}(x), m=1, \cdots, n$, of $p(x)$,

$$
p_{m}(x)=\frac{1}{\sqrt{n}} p\left(\sqrt{n}\left(x-\lambda_{m}\right)\right), \quad 1 \leq m \leq n,
$$

where the supports of all the $p_{m}(x)$, centered at $\lambda_{m}^{\prime} s$ points, $m=1, \cdots, n$, are all disjoint. Observe that $\int_{-\infty}^{\infty} p_{m}(x) d x=\frac{1}{n}$, what make the union

$$
q(x)=\sum_{i=1}^{n} p_{m}(x)
$$

to be also a normalized probability distribution, $\int_{-\infty}^{\infty} q(x) d x=1$. For every $p_{m}(x)$, a straightforward calculation shows that

$$
\begin{aligned}
H\left(p_{m}\right) & =\frac{1}{n} H(p)+\frac{1}{n} \log \sqrt{n} \\
D\left(p_{m}\right) & =\frac{1}{n \sqrt{n}} D(p) .
\end{aligned}
$$

Taking into account that the $m$ replicas are supported on disjoint intervals on $\mathbf{R}$, we obtain

$$
\begin{aligned}
& H(q)=H(p)+\log \sqrt{n}, \\
& D(q)=\frac{1}{\sqrt{n}} D(p) .
\end{aligned}
$$

Then,

$$
\hat{C}(q)=\hat{C}(p),
$$

what completes the proof of the replicant invariance of $\hat{C}$. 


\subsection{Near-Continuity}

Continuity is a desirable property of an indicator of complexity. For a given scale of observation, similar systems should have a similar complexity. In the continuous case, similarity between density functions defined on a common support suggests that they take close values almost everywhere. More strictly speaking, let $\delta$ be a positive real number. It will be said that two density functions $f(x)$ and $g(x)$ defined on the interval $I \in \mathbf{R}$ are $\delta$-neighboring functions on $I$ if the Lebesgue measure of the points $x \in I$ verifying $|f(x)-g(x)| \geq \delta$ is zero. A real map $T$ defined on density functions on $I$ will be called near-continuous if for any $\epsilon>0$ there exists $\delta(\epsilon)>0$ such that if $f(x)$ and $g(x)$ are $\delta$-neighboring functions on $I$ then $|T(f)-T(g)|<\epsilon$.

It can be shown that the information $H$, the disequilibrium $D$ and the shape complexity $\hat{C}$ are near-continuous maps on the space of density functions defined on a compact support. We must stress at this point the importance of the compactness condition of the support in order to have near-continuity. Take, for instance, the density function defined on the interval $[-1, L]$,

$$
g_{\delta, L}(x)=\left\{\begin{array}{cl}
1-\delta & \text { if }-1 \leq x \leq 0 \\
\frac{\delta}{L} & \text { if } 0 \leq x \leq L \\
0 & \text { otherwise }
\end{array}\right.
$$

with $0<\delta<1$ and $L>1$. If we calculate $H$ and $D$ for this distribution 
we obtain

$$
\begin{aligned}
& H\left(g_{\delta, L}\right)=-(1-\delta) \log (1-\delta)-\delta \log \left(\frac{\delta}{L}\right) \\
& D\left(g_{\delta, L}\right)=(1-\delta)^{2}+\frac{\delta^{2}}{L} .
\end{aligned}
$$

Consider also the rectangular density function

$$
\chi_{[-1,0]}(x)= \begin{cases}1 & \text { if }-1 \leq x \leq 0 \\ 0 & \text { otherwise }\end{cases}
$$

If $0<\delta<\bar{\delta}<1, g_{\delta, L}(x)$ and $\chi_{[-1,0]}(x)$ are $\bar{\delta}$-neighboring functions. When $\delta \rightarrow 0$, we have that $\lim _{\delta \rightarrow 0} g_{\delta, L}(x)=\chi_{[-1,0]}(x)$. In this limit process the support is maintained and near-continuity manifests itself as following,

$$
\left[\lim _{\delta \rightarrow 0} \hat{C}\left(g_{\delta, L}\right)\right]=\hat{C}\left(\chi_{[-1,0]}\right)=1
$$

But if we allow the support $L$ to become infinitely large, the compactness condition is not verified and, although $\lim _{L \rightarrow \infty} g_{\delta, L}(x)$ and $\chi_{[-1,0]}(x)$ are $\bar{\delta}$-neighboring distributions, we have that

$$
\left[\left(\lim _{L \rightarrow \infty} \hat{C}\left(g_{\delta, L}\right)\right) \rightarrow \infty\right] \neq \hat{C}\left(\chi_{[-1,0]}\right)=1
$$

Then near-continuity in the map $\hat{C}$ is lost due to the non-compactness of the support when $L \rightarrow \infty$. This example suggests that the shape complexity $\hat{C}$ is near-continuous on compact supports and this property will be rigorously proved elsewhere. 


\subsection{The minimal shape complexity}

If we calculate $\hat{C}$ on the example given by Eq. (13), we can verify that the shape complexity can be as large as wanted. Take, for instance, $\delta=\frac{1}{2}$. The measure $\hat{C}$ reads now

$$
\hat{C}\left(g_{\delta=\frac{1}{2}, L}\right)=\frac{1}{2} \sqrt{L}\left(1+\frac{1}{L}\right)
$$

Thus $\hat{C}$ becomes infinitely large after taking the limits $L \rightarrow 0$ or $L \rightarrow \infty$.

Remark that even in the case $g_{\delta, L}$ has a finite support, $\hat{C}$ is not upper bounded. The density functions, $g_{\left(\delta=\frac{1}{2}\right),(L \rightarrow 0)}$ and $g_{\left(\delta=\frac{1}{2}\right),(L \rightarrow \infty)}$, of infinitely increasing complexity have two zones with different probabilities. In the case $L \rightarrow 0$ there is a narrow zone where probability rises to infinity and in the case $L \rightarrow \infty$ there exists an increasingly large zone where probability tends to zero. Both kind of density functions show a similar pattern to distributions of maximal LMC complexity in the discrete case, where there is an state of dominating probability and the rest of states have the same probability.

The minimal $\hat{C}$ given by Eq. (17) is found when $L=1$, that is, when $g_{\delta, L}$ becomes the rectangular density function $\chi_{[-1,1]}$. In fact, the value $\hat{C}=1$ is the minimum of possible shape complexities and it is reached only on the rectangular distributions. We sketch now some steps that prove this result. 
Suppose

$$
f=\sum_{k=1}^{n} \lambda_{k} \chi_{E_{k}}
$$

to be a density function consisting of several rectangular pieces $E_{k}$, $k=1, \cdots, n$, on disjoint intervals. If $\mu_{k}$ is the Lebesgue measure of $E_{k}$, calculation of $\hat{C}$ gives

$$
\hat{C}(f)=\prod_{k=1}^{n}\left(\lambda_{k}^{-\lambda_{k} \mu_{k}}\right) \cdot\left(\sum_{k=1}^{n} \lambda_{k}^{2} \mu_{k}\right) .
$$

Lagrange multipliers method is used to find the real vector $\left(\mu_{1}, \mu_{2}, \cdots, \mu_{n}\right.$; $\left.\lambda_{1}, \lambda_{2}, \cdots, \lambda_{n}\right)$ that makes extremal the quantity $\hat{C}(f)$ under the condition $\sum_{k=1}^{n} \lambda_{k} \mu_{k}=1$. This is equivalent to studying the extrema of $\log \hat{C}(f)$. We define the function $z\left(\lambda_{k}, \mu_{k}\right)=\log \hat{C}(f)+\alpha\left(\sum_{k=1}^{n} \lambda_{k} \mu_{k}-1\right)$, then

$$
z\left(\lambda_{k}, \mu_{k}\right)=-\sum_{k=1}^{n} \mu_{k} \lambda_{k} \log \lambda_{k}+\log \left(\sum_{k=1}^{n} \mu_{k} \lambda_{k}^{2}\right)+\alpha\left(\sum_{k=1}^{n} \lambda_{k} \mu_{k}-1\right) .
$$

Differentiating this expression and making the result equal to zero we obtain

$$
\begin{aligned}
& \frac{\partial z\left(\lambda_{k}, \mu_{k}\right)}{\partial \lambda_{k}}=-\mu_{k} \log \lambda_{k}-\mu_{k}+\frac{2 \lambda_{k} \mu_{k}}{\sum_{j=1}^{n} \mu_{j} \lambda_{j}^{2}}+\alpha \mu_{k}=0 \\
& \frac{\partial z\left(\lambda_{k}, \mu_{k}\right)}{\partial \mu_{k}}=-\lambda_{k} \log \lambda_{k}+\frac{\lambda_{k}^{2}}{\sum_{j=1}^{n} \mu_{j} \lambda_{j}^{2}}+\alpha \lambda_{k}=0
\end{aligned}
$$

Dividing Eq. (19) by $\mu_{k}$ and Eq. (20) by $\lambda_{k}$ we get

$$
\begin{gathered}
\frac{2 \lambda_{k}}{\sum_{j=1}^{n} \mu_{j} \lambda_{j}^{2}}+\alpha-1=\log \lambda_{k} \\
\frac{\lambda_{k}}{\sum_{j=1}^{n} \mu_{j} \lambda_{j}^{2}}+\alpha=\log \lambda_{k} .
\end{gathered}
$$


Solving these two equations for every $\lambda_{k}$ we have

$$
\lambda_{k}=\sum_{j=1}^{n} \mu_{j} \lambda_{j}^{2} \text { for all } k .
$$

Therefore $f$ is a rectangular function taking the same value $\lambda$ for every interval $E_{k}$, that is, $f$ is the rectangular density function

$$
f=\lambda \cdot \chi_{L} \quad \text { with } \lambda=\frac{1}{\sum_{i=1}^{n} \mu_{i}}=\frac{1}{L}
$$

where $L$ is the Lebesgue measure of the support.

Then $\hat{C}(f)=1$ is the minimal value for a density function composed of several rectangular pieces because, as we know for the example given by Eq. (17), $\hat{C}(f)$ is not upper bounded for this kind of distributions.

Furthermore, for every compactly supported density function $g$ and for every $\epsilon>0$, it can be shown that near-continuity of $\hat{C}$ allows to find a $\delta$-neighboring density function $f$ of the type given by expression (18) verifying $|\hat{C}(f)-\hat{C}(g)|<\epsilon$. The arbitrariness of the election of $\epsilon$ brings us to conclude that $\hat{C}(g) \geq 1$ for every probability distribution $g$. Thus, we can conclude that the minimal value of $\hat{C}$ is 1 and it is reached only by the rectangular density functions. 


\section{Conclusions}

Complexity theory of discrete systems has been equipped with a new function that not only vanishes for perfectly ordered and disordered systems but also has resulted helpful in detecting complexity in patterns produced by a process. Thus LMC complexity has been shown very useful to quantify complex behaviour in local transitions to chaos in discrete mappings [10], it has permitted us to advance the concept of maximum complexity path in the field of systems far from equilibrium [11], and, furthermore, a first attempt to quantify complexity in a model of a two-level laser system was performed in Ref. [12].

Another remarkable feature of LMC complexity is the extension $\hat{C}$ to the continuous case. Results found in the discrete and the continuous case are consistent: extreme values of $\hat{C}$ are observed for distributions characterized by a peak superimposed to a uniform sea. Other merits of this extension have been studied and explained in the present work.

First, we find that this quantity is invariant under translations and rescaling transformations. $\hat{C}$ does not change if the scale of the system is modified but its shape is maintained. It has been calculated on different families of distributions invariant under those transformations. The re-

sult allows us to consider $\hat{C}$ as a new parameter that characterizes every family of distributions. 
Second, it seems reasonable and intuitive that the complexity of $m$ replicas of a given system should be the same to the original one. We show that $\hat{C}$ embodies this property and it is invariant under replication.

Third, continuity is not an evident property for such a map $\hat{C}$. Thus the compactness of the support of the distributions is an important requirement in order to have similar complexity for neighboring distributions. This condition has been strictly established and stood out with an example.

Finally, complexity should be minimal when the system has reached equipartition. We demonstrate that the minimum of $\hat{C}$ is found on the rectangular density functions. Its value is $\hat{C}=1$. Moreover, $\hat{C}$ is not an upper bounded function and it can become infinitely large.

We believe and we hope that the present discussion on the extension of LMC complexity to the continuous case may trigger some practical future considerations in the area of complex systems theory.

Acknowledgements R.G. Catalán acknowledges Spanish DGES for partial financial support (Project PB98-0551).

\section{References}


[1] P. Grassberger, Int. J. Theor. Phys. 25, 907 (1986).

[2] S. Lloyd and H. Pagels, Ann. Phys. (NY) 188, 186 (1988).

[3] J.S. Shiner, M. Davison and P.T. Landsberg, Phys. Rev. E 59, 1459 (1999).

[4] A.N. Kolmogorov, Probl. Inform. Theory 1, 3 (1965).

[5] G. Chaitin, J. Assoc. Comput. Mach. 13, 547 (1966).

[6] A. Lempel and J. Ziv, IEEE Trans. Inform. Theory 22, 75 (1976).

[7] J.P. Crutchfield and K. Young, Phys. Rev. Lett. 63, 105 (1989).

[8] B.A. Huberman and T. Hogg, Physica D 22, 376 (1986).

[9] C. Adami and N.T. Cerf, Physica D 137, 62 (2000).

[10] R. López-Ruiz, H.L. Mancini and X. Calbet, Phys. Lett. A 209, 321 (1995).

[11] X. Calbet and R. López-Ruiz, Phys. Rev. E 63, 066116(9) (2001).

[12] R. López-Ruiz, Int. J. of Bifurcation and Chaos 11, 2669 (2001).

[13] C.Anteneodo and A.R. Plastino, Phys. Lett. A 223, 348 (1996).

[14] C.E. Shannon and W. Weaver, The Mathematical Theory of Communication, University of Illinois Press, Urbana, 1949. 
[15] A.I. Khinchin, Mathematical Foundations of Information Theory, Dover, New York, 1957.

[16] A. Wehrl, Rev. Mod. Phys. 50, 221 (1978).

[17] Y. ZuGuo and C. GuoYi, Commun. Theor. Phys. (Beijing, China) 33, 673 (2000).

[18] F. Gouzhang, S. Songbai and L. Peicheng, Journal of Hydraulic Engineering (Chinese CHES) 11, article 14 (1998).

[19] D.P. Feldman and J.P. Crutchfield, Phys. Lett. A 238, 244 (1998).

[20] C. Tsallis, R.S. Mendes and A.R. Plastino, Physica A 261, 534 (1998). 